\title{
Cutaneous melanoma and the immunotherapy revolution (Review)
}

\author{
GIULIA C. LEONARDI ${ }^{1}$, SAVERIO CANDIDO ${ }^{1,2}$, LUCA FALZONE $^{3}$, \\ DEMETRIOS A. SPANDIDOS ${ }^{4}$ and MASSIMO LIBRA ${ }^{1,2}$ \\ ${ }^{1}$ Department of Biomedical and Biotechnological Sciences; ${ }^{2}$ Research Center for Prevention, \\ Diagnosis and Treatment of Cancer, University of Catania, I-95123 Catania; \\ ${ }^{3}$ Epidemiology Unit, IRCCS Istituto Nazionale Tumori ‘Fondazione G. Pascale', I-80131 Naples, Italy; \\ ${ }^{4}$ Laboratory of Clinical Virology, Medical School, University of Crete, 70013 Heraklion, Greece
}

Received May 22, 2020; Accepted June 23, 2020

DOI: $10.3892 /$ ijo.2020.5088

\begin{abstract}
In a relatively short period of time, treatment strategies for metastatic melanoma have radically changed leading to an unprecedented improvement in patient survival. In this period, immunotherapy options have evolved from cytokine-based approaches to antibody-mediated inhibition of immune checkpoints, cancer vaccines and pharmacological modulation of the melanoma microenvironment. Combination of immunotherapy strategies and the association of immune checkpoint inhibitors (ICIs) with BRAF V600 targeted therapy show encouraging results. The future of drug development in this field is promising. The comprehension of primary and acquired resistance mechanisms to ICIs and the dissection of melanoma immunobiology will be instrumental for the development of new treatment strategies and to improve clinical trial design. Moreover, biomarker discovery will help patient stratification and management during immunotherapy treatment. In this review, we summarize landmark clinical trials of immune checkpoint inhibitors in advanced melanoma and discuss the rational for immunotherapy combinations. Immunotherapy approaches at early stage of clinical development and recent advances in melanoma immunotherapy biomarker development are also discussed.
\end{abstract}

\section{Contents}
1. Introduction
2. CTLA-4 blockade
3. PD-1 blockade

Correspondence to: Professor Massimo Libra, Department of Biomedical and Biotechnological Sciences, University of Catania, Via Santa Sofia 97, I-95123 Catania, Italy

E-mail: m.libra@unict.it

Key words: melanoma, immunotherapy, PD-1, CTLA-4, tumor microenvironment
4. Immune checkpoint inhibitor combinations

5. Talimogene laherparepvec (T-VEC)

6. Other immunotherapy strategies

7. Combination of immune checkpoint inhibitors and targeted therapy

8. Host microbiota and response to immunotherapy

9. Immunotherapy biomarkers

10. Conclusions

\section{Introduction}

Skin cancers are among the most common cancers diagnosed in the United States. The incidence of melanoma of the skin has risen in the last three decades and although melanoma accounts for approximately $1 \%$ of all skin cancers it causes most of the skin cancer deaths (1). When melanoma is diagnosed in its early stages, surgical resection of the lesion is associated with favorable prognosis. However, for locally advanced and metastatic disease surgery is no longer sufficient. The 5-year survival for localized melanoma is $99 \%$, but it is $20 \%$ when distant metastases are present (1).

Melanoma is one of the most sensitive tumors to immune modulation. Several factors may explain melanoma cell susceptibility to immune system activation including high tumor mutational load due to ultraviolet light exposure, expression of cancer testis antigens and mimicry of melanocyte lineage proteins with pathogen-associated antigens (3-5). In this context T-cell response seems to play a central role to keep the melanoma at bay. Tumor infiltrated lymphocytes (TILs) are central to the development of an anti-tumor immune response and a subset of TILs demonstrate cytolytic activity against autologous tumors in melanoma patients (Fig. 1) (6). Their presence also correlates with increased survival and reduced risk of metastasis (6). In the past decades several clinical trials aimed at eliciting T-cell response with local or systemic immunomodulatory drugs such as interferon (IFN)- $\alpha(7,8)$, interleukin (IL)-2 $(9,10)$, cancer vaccines $(11,12)$ and adoptive cell transfer (13). Despite some evidence of activity, these trials failed to demonstrate sustained benefit in metastatic melanoma patients. More recently, immune checkpoint inhibitors (ICIs) 
against cytotoxic T-lymphocyte antigen-4 (CTLA-4) and programmed death-1 (PD-1) have dramatically changed the management of both unresectable and metastatic melanoma as well as those at high risk for recurrence after resection (Table I) (14-16). Unfortunately, primary and secondary resistance and the absence of predictive markers of response are challenging problems with ICIs therapy (17). Combination of immunotherapy strategies aim to improve response and overcome resistance, while biomarker discovery is fundamental for the optimization of patient selection.

Herein, we review and discuss the rational of approved immunotherapy treatments, of preclinical data and ongoing clinical trials of combination strategies for advanced stage and metastatic melanoma. An overview is also provided of biomarker discovery and evidence on the role of gut microbioma in melanoma immunotherapy.

\section{CTLA-4 blockade}

CTLA-4 is an inhibitory checkpoint receptor that blocks T-cell activation and induces immune suppression (Fig. 2) (18). In 1996 Allison and colleagues showed that CTLA-4 blockade could attenuate the growth of several implanted murine tumors (19). In 2011 ipilimumab, a fully human monoclonal antibody (mAb) IgG1 that inhibits the interaction between CTLA-4 and its ligands, was the first ICI approved by the FDA. In previously treated patients with advanced melanoma, ipilimumab improved median overall survival (OS) compared with gp100 peptide vaccine (10.6 vs. 6.4 months) (20). At 3-years, the survival rate was $22 \%$ followed by a plateau of the survival curve for up to 10 years (21). Combination strategies of ipilimumab with IL-2 or Peg-IFN failed to show any improvement over ipilimumab monotherapy $(22,23)$. Tremelimumab, another monoclonal antibody targeting CTLA-4, failed to demonstrate a survival benefit over standard chemotherapy in a phase III clinical trial (24). In the adjuvant setting, a randomized clinical trial in resected stage III patients showed that ipilimumab improves relapse-free survival (RFS) and OS compared to placebo. However, more than $50 \%$ or patients experienced grade 3-4 adverse events with ipilimumab and 5 patients $(1.1 \%)$ died due to immune related adverse events (25).

\section{PD-1 blockade}

PD-1 is an immune checkpoint with a central role in immunopathology and tumor immune surveillance through effector T-cell inhibition (Fig. 2) (18). In 2014, two mAbs targeting PD-1 (nivolumab and pembrolizumab) received FDA approval, becoming first line treatment option in metastatic melanoma. Randomized clinical trials have shown that monotherapy with nivolumab or pembrolizumab is superior to ipilimumab alone (26). Pembrolizumab monotherapy in treatment naïve and previously treated patients showed sustained response rates of $30-40 \%$ (26-28). In treatment of naïve patients pembrolizumab showed a 3 -year OS rate of $51 \%$ and a 5-year rate of $41 \%(29,30)$. Clinical trials of nivolumab monotherapy provided durable response rate of $32 \%$ in untreated patients and $40 \%$ in previously treated melanoma $(16,31)$. Three-year survival rate for nivolumab in previously untreated patients is $42 \%$ (32) while 5-year survival rate in previously treated patients with nivolumab monotherapy is $35 \%$ (15). Cross study comparisons of homogeneous groups of patients treated with pembrolizumab or nivolumab monotherapy have similar results regarding clinical endpoints and adverse event rates (33). At this time, no good predictive biomarkers for anti-PD-1 mAbs are available and clinical benefit is reached regardless of PD-L1 status (32). Since 2017, adjuvant immunotherapy with single agent anti-PD-1 mAb is the first treatment option in patients with resected stage III disease. Nivolumab improved RFS compared to ipilimumab with lower toxicities (34). In a phase 3 double-blind trial pembrolizumab treatment resulted in significantly longer RFS than placebo with no new toxicities compared to other pembrolizumab monotherapy trials (35).

\section{Immune checkpoint inhibitor combinations}

Monotherapy with ICIs is associated with significant improvement in patient survival, however, response rates are low. With the attempt to increase the number of patients who benefit from ICI therapy, combination of anti-CTLA-4 $\mathrm{mAb}$ plus anti-PD-1 mAb have been evaluated in prospective clinical trials. In particular, two randomized trials showed that the combination of anti-CTLA-4 plus anti-PD-1 mAbs results in increased clinical benefit compared to single agent ipilimumab or nivolumab. The Checkmate-067, a phase 3 randomized clinical trial compared ipilimumab plus nivolumab to nivolumab alone and ipilimumab alone in unresectable/metastatic melanoma. Response rates were 57.6, 43.7 and 19\%, respectively, and 5-year survival rates were $52 \%$ in the combination arm, $44 \%$ in the nivolumab group and $26 \%$ in the ipilimumab arm $(36,37)$. The combination arm showed increased toxicity compared to each monotherapy treatment arm. Specifically, treatment-related adverse events of any grade occurred in $95 \%$ of patients in the combination treatment group compared to $82 \%$ in the nivolumab arm and $86 \%$ in the ipilimumab group. Grade 3 or 4 treatment related adverse events occurred in $55 \%$ of the patients in the nivolumab plus ipilimumab group, $16.3 \%$ of the patients in the nivolumab group and $27.3 \%$ of those in the ipilimumab group (36). In 2015 the FDA approved the combination ipilimumab plus nivolumab on the basis of overall response rate and progression-free survival (PFS) improvement. In an attempt of reducing the toxicity burden of the combination, different dosing schedule by reducing ipilimumab dose and keep more standard dose anti-PD-1 single agents were investigated. Although the ORR appears to be conserved, the expected difference in terms of efficacy and safety are small and only results from larger trial will be conclusive $(38,39)$. In patients with primary or secondary resistance to single agent PD-1 mAbs, ICIs combination or ipilimumab alone represent potential treatment strategies $(40,41)$. Currently, the respective benefits of combination immunotherapy versus sequential immunotherapy are not yet fully understood and is object of an open debate in the clinical and scientific community. On one hand combination strategies are associated with increased adverse events that can be justified by the intent of gaining long-term disease response. On the other hand, the subset of patients who benefit more from the combination is unknow potentially exposing patients to unnecessary toxicities (42). 


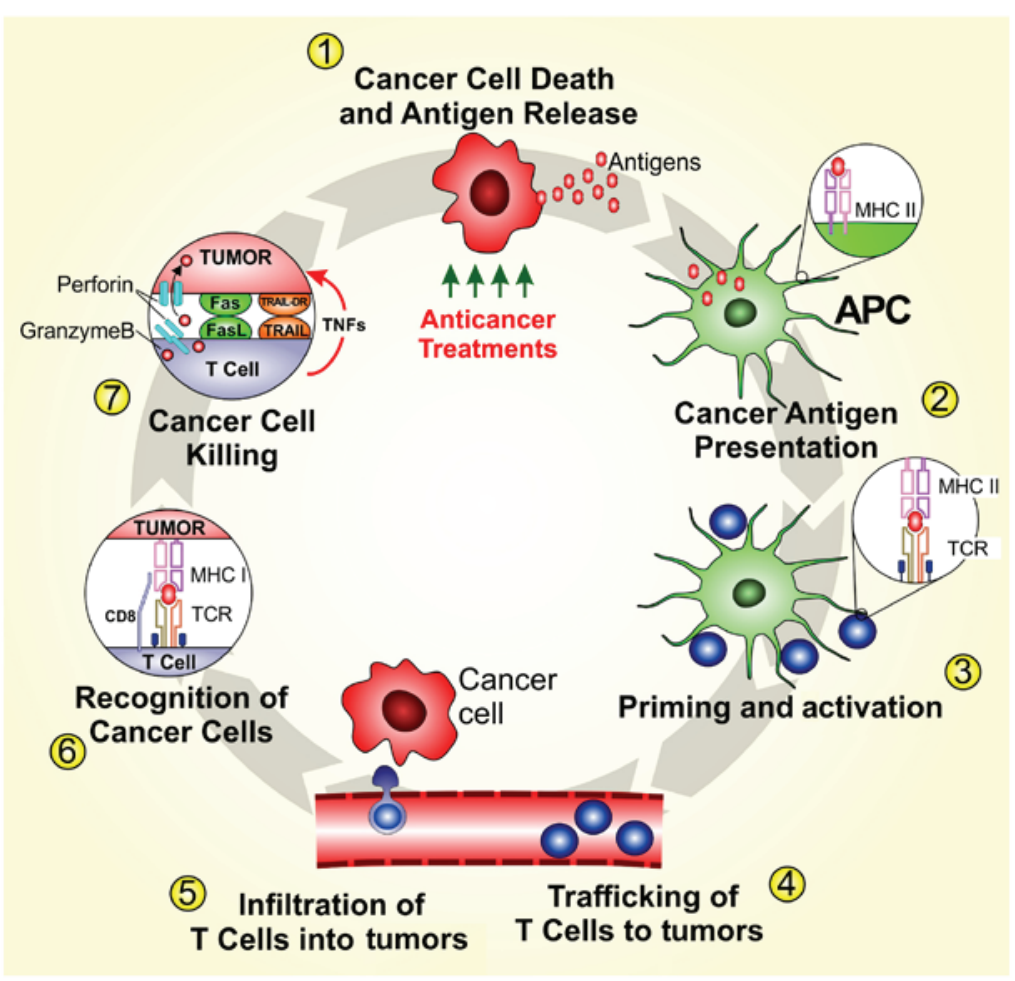

Figure 1 . The cancer immunity cycle.

Brain metastases are a common cause of disabling neurologic complications and poor prognosis in patients with metastatic melanoma. The phase 2 clinical trial CheckMate-204 enrolled patients with small, untreated and asymptomatic brain metastasis and showed that ipilimumab plus nivolumab have clinically meaningful intracranial efficacy (56\% of intracranial response). The safety profile was similar to those reported for the combination in patients without brain metastasis (43). Another phase 2 clinical trial compared the combination of nivolumab plus ipilimumab versus nivolumab alone. Despite the small sample size, ICIs combination was superior to nivolumab monotherapy with a higher proportion of patients achieving intracranial response (44).

No data are yet available on the potential benefit of anti-CTLA-4 plus anti-PD-1 combination in the adjuvant setting. Results from the clinical trial CheckMate-915 (NCT03068455) that compare nivolumab monotherapy to nivolumab plus ipilimumab in resected stage III melanoma are awaited.

\section{Talimogene laherparepvec (T-VEC)}

T-VEC is a type I herpes simplex virus genetically modified to preferentially replicate in tumor cells, enhance antigen loading of MHC class I and express granulocyte-macrophage colony-stimulating factor (GM-CSF) to increase tumor antigen presentation by dendritic cells (DCs) (45). In 2015 T-VEC received approval by the FDA for advanced melanoma. A phase 3 trial in unresected stage IIIB-IV melanoma showed that intratumoral administration of T-VEC improved response rate compared with GM-CSF (26 vs. 6\%). Few responses were obtained in distant non-injected lesions (mainly lung and visceral sites) while the majority of responses were limited to the site of injection and regional non-injected lesions (46). The combination of T-VEC and ICIs has shown interesting results. The phase II study of T-VEC plus ipilimumab versus ipilimumab alone in patients with advanced melanoma showed an improvement in ORR (39 vs. 18\%, respectively) (47). In the phase Ib trial evaluating the association of T-VEC plus pembrolizumab, confirmed objective response rate was $62 \%$, with a complete response rate of $33 \%$ per immune-related response criteria (48). The results of the phase III trial MASTERKEY-265/KEYNOTE-034 of T-VEC plus pembrolizumab compared with pembrolizumab alone are awaited (NCT02263508).

\section{Other immunotherapy strategies}

Despite the impressive results obtained with currently approved ICI treatments strategies, primary and secondary resistance represent major clinical challenges. Several promising immunomodulatory targets have been included in ongoing clinical trials mainly in association with approved anti-PD-1 and anti-CTLA-4 mAbs (Fig. 2).

Inhibitors of lymphocyte activation gene-3 (LAG-3) and T-cell immunoglobulin and mucin-domain containing-3 (TIM-3) in clinical development.LAG-3 is an immune checkpoint receptor found on the cell surface of effector $\mathrm{T}$ cells and regulatory $\mathrm{T}$ cells. It is an inhibitor regulator of $\mathrm{T}$ cell response, activation and growth (49). Relatlimab, LAG525, and MK-4280 are mAbs that targets LAG-3 and are currently under investigation in combination with pembrolizumab or nivolumab (50). While the inhibition of LAG-3 alone has minor effect on T-cell reactivation, the combination with anti-PD-1 $\mathrm{mAb}$ has shown encouraging results $(51,52)$. 
Table I. Landmark clinical trials of immunotherapy in locally advanced and metastatic melanoma.

\begin{tabular}{|c|c|c|c|c|c|c|}
\hline Trial name & $\begin{array}{l}\text { Primary } \\
\text { outcome }\end{array}$ & Treatment arms & ORR $(\%)$ & $\begin{array}{l}\text { Median PFS } \\
\text { (months) }\end{array}$ & $\begin{array}{l}\text { Median OS } \\
\text { (months) }\end{array}$ & $\begin{array}{c}\text { 1yr-RFS } \\
(\%)\end{array}$ \\
\hline \multirow[t]{3}{*}{ CA184-002 (20) } & \multirow[t]{3}{*}{ OS } & gp100 vaccine & 1.5 & 2.8 & 6.4 & - \\
\hline & & gp100 vaccine+ipilimumab & 5.7 & 2.8 & 10.0 & - \\
\hline & & ipilimumab & 11.0 & 2.9 & 10.1 & - \\
\hline \multirow[t]{2}{*}{ CA184-024 } & \multirow[t]{2}{*}{ OS } & Dacarbazine & 10.3 & 3.0 & 9.1 & - \\
\hline & & Dacarbazine+ipilimumab & 15.2 & 3.0 & 11.2 & - \\
\hline \multirow[t]{2}{*}{ CheckMate 066 (32) } & \multirow[t]{2}{*}{ OS } & Dacarbazine & 14.4 & 2.2 & 11.2 & - \\
\hline & & Nivolumab & 42.9 & 5.1 & 37.5 & - \\
\hline \multirow[t]{3}{*}{ KEYNOTE-006 $(28,30)$} & \multirow[t]{3}{*}{ PFS, OS } & Ipilimumab & 11.9 & 3.4 & 16.0 & - \\
\hline & & Pembrolizumab q2w & 33.7 & 5.6 & $32.7^{\mathrm{a}}$ & - \\
\hline & & Pembrolizumab q3w & 32.9 & 4.1 & & \\
\hline \multirow[t]{3}{*}{ CheckMate 067 (37) } & \multirow[t]{3}{*}{ PFS, OS } & Ipilimumab & 19.0 & 2.9 & 19.9 & - \\
\hline & & Nivolumab & 45.0 & 6.9 & 36.9 & - \\
\hline & & Nivolumab+ipilimumab & 58.0 & 11.5 & NR & - \\
\hline \multirow[t]{2}{*}{ OPTiM (46) } & \multirow{2}{*}{$\begin{array}{l}\text { Durable response } \\
\text { lasting } \geq 6 \text { months }\end{array}$} & GM-CSF & Not reported & Not reported & 18.9 & - \\
\hline & & $\mathrm{T}-\mathrm{VEC}$ & Not reported & Not reported & 23.3 & - \\
\hline \multirow[t]{2}{*}{ CheckMate 238 (34) } & \multirow[t]{2}{*}{ RFS } & Nivolumab & - & - & - & 70.5 \\
\hline & & Ipilimumab & - & - & - & 60.8 \\
\hline EORTC1325/ & \multirow[t]{2}{*}{ RFS } & Pembrolizumab & - & - & - & 75.4 \\
\hline KEYNOTE-054 (35) & & Placebo & - & - & - & 61.0 \\
\hline
\end{tabular}

ORR, overall response rate; OS, overall survival; PFS, progression-free survival; RFS, relapse-free survival; NR, not reached; q2w, every two

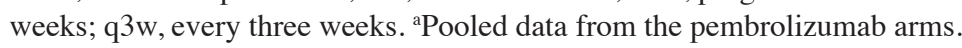

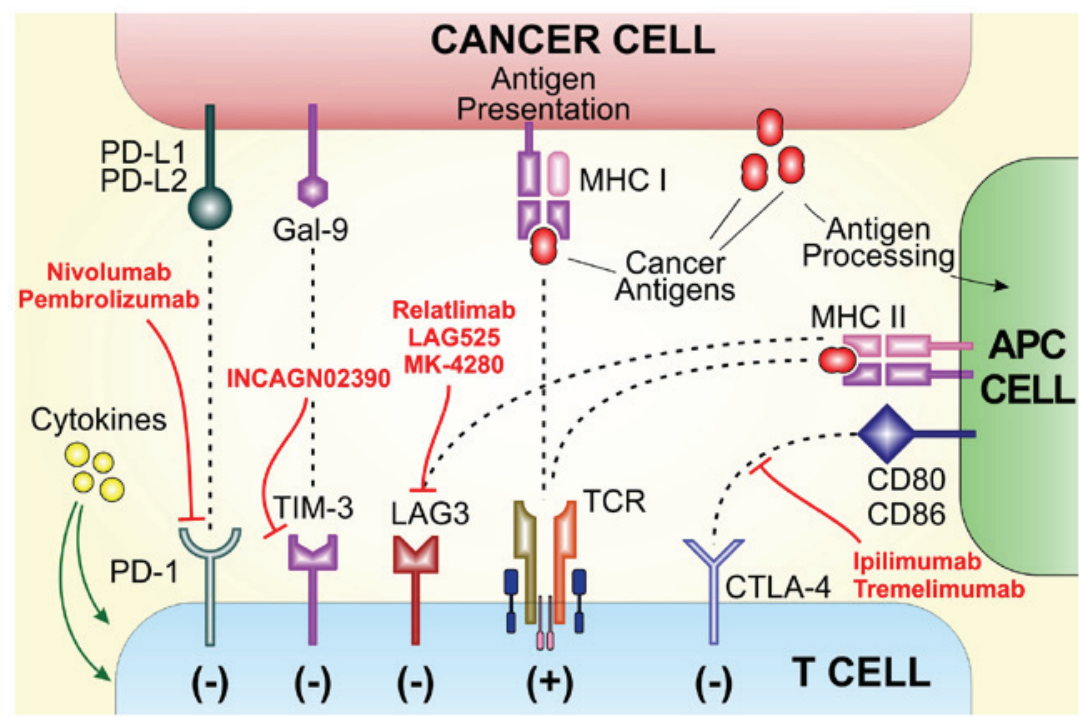

Figure 2. Immune checkpoints and their inhibitors in advanced melanoma. PD, programmed death; TIM-3, T-cell immunoglobulin and mucin-domain containing-3; LAG3, lymphocyte activation gene 3; TCR, T cell receptor; CTLA-4, cytotoxic T-lymphocyte antigen-4.

TIM-3 is an immune checkpoint expressed on IFN- $\gamma$-producing T cells, FoxP $3^{+}$Treg cells and macrophages and DCs where it suppresses their responses upon interaction with their ligands. In vivo blockade of TIM-3 with other check-point inhibitors enhances anti-tumor immunity and suppresses tumor growth in several preclinical tumor models (53). INCAGN02390 is a TIM-3 inhibitor that is undergoing trial for the treatment of advanced solid tumor including melanoma (NCT03652077).

IDO inhibitors. Indoleamine 2,3-dioxygenase 1 (IDO1) is an enzyme involved in tryptophan catabolism with a 
central immunosuppressive function within the tumor microenvironment (54). Several IDO inhibitors (indiximod, epacadostat and BMS-986205) are currently evaluated in clinical trials in association with pembrolizumab, nivolumab or ipilimumab (54). Regrettably, the phase III clinical trial ECHO-301/KEYNOTE-252 in advanced melanoma failed to demonstrate PFS benefit in the arm of pembrolizumab with epcadostat compared to pembrolizumab alone (55).

Cytokines. Cytokines are the first class of immunomodulatory agents that have found clinical application in melanoma. Indeed, IL-2 and IFN- $\alpha$ are both FDA approved for adjuvant treatment in melanoma $(7,56)$. Other cytokines such as IL-12, IL-15, IL-18, IL-21 and GM-CSF have shown interesting results in preclinical and clinical settings. However, single agent cytokine strategy does not appear feasible due to their pleiotropic activity and the critical toxicity profile especially at high dose (56). With this in mind, NTRK-214 is a prodrug of conjugated IL-2, retaining the same amino acid sequence as human recombinant IL-2. The IL-2 core is conjugated to 6 releasable polyethylene glycol (PEG) chains that in vivo slowly release generating active IL-2 conjugates (57). An ongoing phase I/II clinical trial aims to evaluate the tolerability and efficacy of NTRK-214 with nivolumab and ipilimumab plus nivolumab (NCT02983045).

Modulation of the tumor microenvironment and the innate immune system. Tilsotolimod is a synthetic TLR-9 agonist oligonucleotide that acts on macrophages and DCs and can stimulate antigen presentation and $\mathrm{T}$ cell activation and proliferation. Intratumoral tilsotolimod in combination with ipilimumab in PD-1 inhibitor refractory metastatic melanoma is well tolerated and shows significant clinical benefit (ORR $38 \%$ ) and durable response (58). These favorable results have led to an ongoing phase III study of tilsotolimod plus ipilimumab versus ipilimumab alone (NCT03445533). Another intratumoral TLR-9 agonist, SD-101, is in clinical development. The phase Ib/II clinical trial SYNERGY-001/KEYNOTE-184 evaluates the combination of SD-101 and pembrolizumab in patients with unresectable stage IIIC-IV melanoma and naïve to PD-1 axis inhibitors. Preliminary results show that the combination is well-tolerated, with promising high response rates and PFS (59). CD40 is expressed on macrophages and other antigen-presenting cells and its agonists stimulate maturation and increase macrophage killing activity against tumor cells (60). On the other hand, tumor-associated macrophages can be characterized by tumor-promoting phenotype (61). This phenotype is a consequence, among other factors, of the continuous activation of the colony-stimulating factor-1 (CSF-1) axis (62). An ongoing phase I/Ib trial is evaluating the safety and efficacy of the CSF-1 receptor inhibitor, cabiralizumab, combined with the CD40 agonist, APX005M, with or without nivolumab in patients with advanced melanoma (NCT03502330).

Vaccines. Therapeutic cancer vaccines aim at inducing a specific immune response against tumor antigens. In melanoma patients, peptide vaccines have been tested in association with ipilimumab, but failed to demonstrate an advantage compared to ipilimumab alone $(20,63)$. In a phase I clinical trial, tremelimumab plus MART-1 peptide-pulsed DCs resulted in objective and durable tumor responses compared to each agent alone (64). A phase I trial in patients with pretreated advanced melanoma showed that autologous monocyte-derived DCs electroporated with synthetic mRNA (TriMixDC-MEL) are immunogenic and have antitumor activity (65). TriMixDC-MEL combined with ipilimumab has shown $38 \%$ of durable tumor responses in a phase II trial (66). The tumor lysate, particle-loaded, dendritic cell (TLPLDC) vaccine uses yeast cell wall particles to load tumor lysate into autologous DCs. The phase IIb trial of TLPLDC vs. placebo in resected stage III/IV patients showed an increased 24-month DFS. The trial showed also a potential synergistic effect of TLPLDC plus ipilimumab to be confirmed in a phase III study evaluating adjuvant TLPLDC plus ipilimumab versus ipilimumab alone in resected stage IV patients (67).

Adoptive $T$ cell transfer. The presence of tumor-reactive $\mathrm{T}$ cells has been associated with the success of ICIs $(68,69)$. When patients do not have functional tumor-antigen-specific $\mathrm{T}$ cells with high-affinity $\mathrm{T}$ cell receptors (TCRs), $\mathrm{T}$ cell therapies can transfer such $\mathrm{T}$ cell populations by either expanding pre-existing anti-tumor $\mathrm{T}$ cells or by using gene-therapy to alter T cells to become melanoma-specific with a high-affinity TCR (70). ACT of autologous tumor infiltrating lymphocytes with high-dose IL-2 was the first clinical trial to show that tumor-reactive $\mathrm{T}$ cells could mediate melanoma regression (71). However, TIL has not been approved as anti-cancer treatment yet due to lack of results from sufficiently powered prospective randomized clinical trials $(13,72)$. In order to improve clinical benefit and survival, TIL therapy could be combined with other immunotherapies (70). ACT through chimeric antigen receptor (CAR) T-cell therapy has been successful in hematological malignancies. However, less response was seen in the treatment of solid tumors such as melanoma (73). Combination of CAR-T therapy and immune checkpoint blockade, targeted therapy might induce desired clinical responses (73). The adoptive transfer of autologous $\mathrm{T}$ cells transduced with a retrovirus encoding a TCR against an HLA-A*0201 restricted NY-ESO-1 epitope is a potentially effective treatment for some refractory metastatic melanoma patients (74).

\section{Combination of immune checkpoint inhibitors and targeted therapy}

Targeted therapy and ICIs have radically changed the management of different tumor types, including advanced stage melanoma (75). However, both approaches have limitations, including limited duration of response with targeted therapy and low overall response rate without clear predictive biomarkers in patients treated with ICIs. Therefore, great interest has been shown on the possibility of combination strategies that could take advantage from the high response rate of targeted therapy with the long-term disease control of ICIs. Despite some contrasting preclinical results that have been observed on the association of BRAF and MEK inhibitors with immunotherapy, various trials are ongoing investigating the association of MAPK inhibitors with ICIs and other immunotherapy strategies (76). Several trials with CTLA-4 
inhibitors and MAPK inhibitors have raised concerns due to the toxicities associated with the combinations that led to early trial termination $(77,78)$. More tolerable and with good disease control rates seem to be the association of PD-1/PD-L1 axis inhibitors with BRAF and MEK inhibitors (79). In this context, study design is fundamental to properly conduct clinical trial of combination strategies without increasing toxicities. The ongoing phase III clinical trial DREAMseq (NCT02224781) studies how well initial treatment works with ipilimumab and nivolumab followed by dabrafenib and trametinib, and compares it to initial treatment with dabrafenib and trametinib followed by ipilimumab and nivolumab in treating patients with stage III-IV BRAF V600 melanoma.

\section{Host microbiota and response to immunotherapy}

The improvement of clinical efficacy of immunotherapy strategies is a central goal of translational and clinical research in immuno-oncology. In recent years, several factors have been shown to influence the immune response during ICI treatment (80-83). In this context, the human microbiota seems to be an important modulator of the immune system response in different physiopathological conditions, including cancer development and response to anti-cancer treatment (84). In a melanoma mouse model, different commensal gut microbiota composition was associated with difference in melanoma growth and spontaneous antitumor immunity which were eliminated upon cohousing or after fecal transfer (85). In a RET melanoma mouse model with dysbiosis induced by antibiotic treatment, gut colonization with Akkermansia muciniphila restored responsiveness to $\mathrm{PD}-1$ blockade compared to control and increased the recruitment of $\mathrm{CD}^{+} \mathrm{T}$ lymphocytes into mouse tumor beds (86). In 112 melanoma patients treated with anti-PD-1 mAbs, significant differences were observed in the gut microbiome of responders versus non-responders. Analysis of patient fecal microbiome samples showed significantly higher species diversity and relative abundance of the Ruminococcaceae family in responding patients. Moreover, mice receiving fecal microbiota transplantation (FMT) with stool from responders to PD-1 inhibitors also exhibited improved response to anti-PD-L1 therapy compared to mice transplanted with stool from melanoma patients that did not respond to anti-PD-L1 therapy (87). Several preclinical and clinical works show the relevance of the modulation of the gut microbiota in ICIs efficacy (88-91). Taken together these data sustain potential use of the human microbiome as a predictive biomarker of response to ICIs. Moreover, the modulation of specific component of the human microbiome by FMT or oral supplementation through probiotics may improve response rates and other clinical endpoints of ICIs therapy in cancer patients. Prospective randomized clinical trials are awaited to better understand the impact of such approaches.

\section{Immunotherapy biomarkers}

Targeted therapy derives its efficacy from the presence of a specific tumor feature, such as the BRAF V600 mutation, that drives tumor growth and that represent a specific biomarker of response to the drug targeting the aberrant pathway. In melanoma, primary and secondary resistance to targeted therapy are challenging problems and several studies have tried to improve BRAF V600 detection of prognostic and predictive markers (92-94). Despite great efforts, melanoma immunotherapy and especially ICIs, which are already approved for clinical use, lack biomarker response. This is especially urgent due to the relatively low response rate of immunotherapy. Regarding inhibitors of PD-1/PD-L1 axis, PD-L1 expression assessed by immunohistochemistry (IHC) staining has been used as biomarkers in several clinical trials. Different PD-L1 IHC antibodies with non-homogeneous cut-off values among studies have generated contrasting results on the role of PD-L1 for patient stratification $(26,31,36)$. Although PD-L1 status is not currently considered a valid stratification marker, it warrants further analysis since it may indicate underlying biological insights (95). More comprehensive models are under investigation to better characterize the tumor microenvironment and define predictive biomarkers for immunotherapy. In this context an important study by Tumeh and collaborators (96) has shown that the presence of $\mathrm{CD} 8^{+} \mathrm{T}$ cells that cause upregulation of PD-L1 expression on melanoma cells at the invasive tumor margin might more clearly explain primary resistance or responsiveness to anti-PD-1 mAbs. Gene-expression profile has been suggested as a marker of response (97). IFN $\gamma$ secreted by $\mathrm{CD}^{+} \mathrm{T}$ cells is the principal mediator of intratumoural antitumor inflammation, a gene expression profile termed 'T-cell-inflamed tumor' has been associated with response to diverse immunotherapies including IL-2, vaccines, ICIs and cancer vaccine $(98,99)$. Moreover, primary and secondary resistance to PD-1/PD-L1 pathway inhibitors is associated with a low IFN $\gamma$ gene expression signature that can be mediated by activation of PTEN and WNT/ $\beta$-catenin pathway, impairment of JAK2 signaling or alteration of antigen presentation through structural or functional impairment of MHC class I mediated antigen presentation (100-103). A study with the largest whole exome sequencing and transcriptome sequencing analysis of tumor material from patients with metastatic melanoma receiving immune-checkpoint inhibitors has been published (104). The study supports the correlations between baseline immune infiltrate and treatment response, but also show inconsistent associations of tumor mutational burden, and prove that multiple novel genomic and transcriptomic features predict selective response, including features associated with MHC-I and MHC-II antigen presentation (104). Moreover, the authors constructed predictive models integrating clinical, genomic and transcriptomic characteristics to identify patients with melanoma with intrinsic resistance to anti-PD1 $\mathrm{mAb}$ (104). Recently, a growing body of evidence is trying to correlate ICI efficacy to the expression levels of PD-L1 detected in circulating tumor DNA $(105,106)$. Finally, studies have proposed the analysis of matrix metalloproteinase (MMPs), known to be strictly involved in melanoma growth $(107,108)$, as good indicator of response to immunotherapy. Moogk and colleagues (109) showed an inverse association between anti-tumor T-cell response and MMP-23 expression in primary melanoma tumors treated with adjuvant immunotherapy. The authors concluded that MMP-23 expression is associated with shorter periods of PFS and therefore may represent a potential therapeutic target in melanoma, as well as a possible biomarker for evaluating response of melanoma patients to immunotherapies. 


\section{Conclusions}

The number of effective treatments for patients with metastatic melanoma have increased rapidly in recent few years. In this scenario, immunotherapy combinations and immunotherapy plus targeted therapy hold great expectation. Moreover, results from the clinical trial DREAMseq are eagerly awaited and will shed light on the best sequential treatment option in metastatic melanoma patients with BRAF V600E. Given the complexity of the antitumor immune response, the identification of biomarkers of response and the mechanisms associated with primary and secondary resistance are of utmost importance. These insights will allow the next generation of immunotherapy approaches that will be tailored on specific melanoma genomic features and its tumor microenvironment characteristics.

\section{Acknowledgments}

Not applicable.

\section{Funding}

This study was partially supported by the Italian League against Cancer (LILT).

\section{Availability of data and materials}

Not applicable

\section{Authors' contributions}

GCL conceived the work, performed bibliographic research and wrote the manuscript, SC prepared the figures and provided critical revisions, LF performed bibliographic research and provided critical revisions, DAS, ML contributed to the conception of the work and provided critical revisions. All authors agreed on the final version of the manuscript.

\section{Ethics approval and consent to participate}

Not applicable

\section{Patient consent for publication}

Not applicable

\section{Competing interest}

GCL, SC, LF, ML no competing interest to declare, DAS is the Editor-in-Chief for the journal, but had no personal involvement in the reviewing process, or any influence in terms of adjudicating on the final decision, for this article.

\section{References}

1. Siegel RL, Miller KD and Jemal A: Cancer statistics, 2018. CA Cancer J Clin 68: 7-30, 2018.

2. Leonardi GC, Falzone L, Salemi R, Zanghì A, Spandidos DA, McCubrey JA, Candido S and Libra M: Cutaneous melanoma: From pathogenesis to therapy (Review). Int J Oncol 52: 1071-1080, 2018.
3. Candido S, Rapisarda V, Marconi A, Malaponte G, Bevelacqua V, Gangemi P, Scalisi A, McCubrey JA, Maestro R, Spandidos DA, et al: Analysis of the B-RafV600E mutation in cutaneous melanoma patients with occupational sun exposure. Oncol Rep 31: 1079-1082, 2014.

4. Kawakami Y and Rosenberg SA: T-cell recognition of self peptides as tumor rejection antigens. Immunol Res 15: 179-190, 1996.

5. Faramarzi S and Ghafouri-Fard S: Melanoma: A prototype of cancer-testis antigen-expressing malignancies. Immunotherapy 9: 1103-1113, 2017

6. LeeN,ZakkaLR,Mihm MC Jrand Schatton T: Tumour-infiltrating lymphocytes in melanoma prognosis and cancer immunotherapy. Pathology 48: 177-187, 2016.

7. Kirkwood JM, Ibrahim JG, Sosman JA, Sondak VK, Agarwala SS, Ernstoff MS and Rao U: High-dose interferon alfa- $2 \mathrm{~b}$ significantly prolongs relapse-free and overall survival compared with the GM2-KLH/QS-21 vaccine in patients with resected stage IIB-III melanoma: Results of intergroup trial E1694/S9512/C509801. J Clin Oncol 19: 2370-2380, 2001.

8. Wheatley K, Ives N, Hancock B, Gore M, Eggermont A and Suciu S: Does adjuvant interferon-alpha for high-risk melanoma provide a worthwhile benefit? A meta-analysis of the randomised trials. Cancer Treat Rev 29: 241-252, 2003.

9. Atkins MB, Kunkel L, Sznol M and Rosenberg SA: High-dose recombinant interleukin-2 therapy in patients with metastatic melanoma: Long-term survival update. Cancer J Sci Am 6 (Suppl 1): S11-S14, 2000.

10. Rosenberg SA: IL-2: The first effective immunotherapy for human cancer. J Immunol 192: 5451-5458, 2014.

11. Klebanoff CA, Acquavella N, Yu Z and Restifo NP: Therapeutic cancer vaccines: Are we there yet? Immunol Rev 239: 27-44, 2011.

12. Schwartzentruber DJ, Lawson DH, Richards JM, Conry RM, Miller DM, Treisman J, Gailani F, Riley L, Conlon K, Pockaj B, et al: gp100 peptide vaccine and interleukin-2 in patients with advanced melanoma. N Engl J Med 364: 2119-2127, 2011.

13. Rohaan MW, van den Berg JH, Kvistborg P and Haanen JBAG: Adoptive transfer of tumor-infiltrating lymphocytes in melanoma: A viable treatment option. J Immunother Cancer 6: 102, 2018.

14. Ascierto PA, McArthur GA, Dréno B, Atkinson V, Liszkay G, Di Giacomo AM, Mandalà M, Demidov L, Stroyakovskiy D, Thomas L, et al: Cobimetinib combined with vemurafenib in advanced BRAF(V600)-mutant melanoma (coBRIM): Updated efficacy results from a randomised, double-blind, phase 3 trial. Lancet Oncol 17: 1248-1260, 2016.

15. Hodi FS, Kluger H, Sznol M, Carvajal R, Lawrence D, Atkins M, Powderly J, Sharfman W, Puzanov I, et al: Durable, long-term survival in previously treated patients with advanced melanoma (MEL) who received nivolumab (NIVO) monotherapy in a phase I trial. Cancer Res 76: CT001, 2016.

16. Weber JS, D'Angelo SP, Minor D, Hodi FS, Gutzmer R, Neyns B, Hoeller C, Khushalani NI, Miller WH Jr, Lao CD, et al: Nivolumab versus chemotherapy in patients with advanced melanoma who progressed after anti-CTLA-4 treatment (CheckMate 037): A randomised, controlled, open-label, phase 3 trial. Lancet Oncol 16: 375-384, 2015.

17. Christofi T, Baritaki S, Falzone L, Libra M and Zaravinos A: Current perspectives in cancer immunotherapy. Cancers (Basel) 11: 1472, 2019

18. Wei SC, Duffy CR and Allison JP: Fundamental mechanisms of immune checkpoint blockade therapy. Cancer Discov 8: 1069-1086, 2018.

19. Leach DR, Krummel MF and Allison JP: Enhancement of antitumor immunity by CTLA-4 blockade. Science 271: 1734-1736, 1996

20. Hodi FS, O'Day SJ, McDermott DF, Weber RW, Sosman JA, Haanen JB, Gonzalez R, Robert C, Schadendorf D, Hassel JC, et al: Improved survival with ipilimumab in patients with metastatic melanoma. N Engl J Med 363: 711-723, 2010.

21. Schadendorf D, Hodi FS, Robert C, Weber JS, Margolin K, Hamid O, Patt D, Chen TT, Berman DM and Wolchok JD: Pooled analysis of long-term survival data from phase II and phase III trials of ipilimumab in unresectable or metastatic melanoma. J Clin Oncol 33: 1889-1894, 2015.

22. Weide B, Martens A, Wistuba-Hamprecht K, Zelba H, Maier L, Lipp HP, Klumpp BD, Soffel D, Eigentler TK and Garbe C: Combined treatment with ipilimumab and intratumoral interleukin-2 in pretreated patients with stage IV melanoma-safety and efficacy in a phase II study. Cancer Immunol Immunother 66: 441-449, 2017. 
23. Brohl AS, Khushalani NI, Eroglu Z, Markowitz J, Thapa R, Chen YA, Kudchadkar R and Weber JS: A phase IB study of ipilimumab with peginterferon alfa- $2 \mathrm{~b}$ in patients with unresectable melanoma. J Immunother Cancer 4: 85, 2016.

24. Ribas A, Kefford R, Marshall MA, Punt CJ, Haanen JB, Marmol M, Garbe C, Gogas H, Schachter J, Linette G, et al: Phase III randomized clinical trial comparing tremelimumab with standard-of-care chemotherapy in patients with advanced melanoma. J Clin Oncol 31: 616-622, 2013.

25. Eggermont AM, Chiarion-Sileni V, Grob JJ, Dummer R, Wolchok JD, Schmidt H, Hamid O, Robert C, Ascierto PA, Richards JM, et al: Prolonged survival in stage III melanoma with ipilimumab adjuvant therapy. N Engl J Med 375: 1845-1855, 2016.

26. Robert C, Schachter J, Long GV, Arance A, Grob JJ, Mortier L, Daud A, Carlino MS, McNeil C, Lotem M, et al; KEYNOTE-006 investigators: Pembrolizumab versus ipilimumab in advanced melanoma. N Engl J Med 372: 2521-2532, 2015.

27. Ribas A, Hamid O, Daud A, Hodi FS, Wolchok JD, Kefford R, Joshua AM, Patnaik A, Hwu WJ, Weber JS, et al: Association of pembrolizumab with tumor response and survival among patients with advanced melanoma. JAMA 315: 1600-1609, 2016.

28. Schachter J, Ribas A, Long GV, Arance A, Grob JJ, Mortier L, Daud A, Carlino MS, McNeil C, Lotem M, et al: Pembrolizumab versus ipilimumab for advanced melanoma: Final overall survival results of a multicentre, randomised, open-label phase 3 study (KEYNOTE-006). Lancet 390: 1853-1862, 2017.

29. Hamid O, Robert C, Daud A, Hodi FS, Hwu WJ, Kefford R, Wolchok JD, Hersey P, Joseph R, Weber JS, et al: Five-year survival outcomes for patients with advanced melanoma treated with pembrolizumab in KEYNOTE-001. Ann Oncol 30: $582-588,2019$.

30. Robert C, Ribas A, Schachter J, Arance A, Grob JJ, Mortier L, Daud A, Carlino MS, McNeil CM, Lotem M, et al: Pembrolizumab versus ipilimumab in advanced melanoma (KEYNOTE-006): Post-hoc 5-year results from an open-label, multicentre, randomised, controlled, phase 3 study. Lancet Oncol 20: 1239-1251, 2019.

31. Robert C, Long GV, Brady B, Dutriaux C, Maio M, Mortier L, Hassel JC, Rutkowski P, McNeil C, Kalinka-Warzocha E, et al: Nivolumab in previously untreated melanoma without BRAF mutation. N Engl J Med 372: 320-330, 2015.

32. Ascierto PA, Long GV, Robert C, Brady B, Dutriaux C, Di Giacomo AM, Mortier L, Hassel JC, Rutkowski P, McNeil C, et al: Survival outcomes in patients with previously untreated BRAF wild-type advanced melanoma treated with nivolumab therapy: Three-year follow-up of a randomized phase 3 trial. JAMA Oncol 5: 187-194, 2019

33. Weiss SA, Wolchok JD and Sznol M: Immunotherapy of melanoma: Facts and hopes. Clin Cancer Res 25: 5191-5201, 2019.

34. Weber J, Mandala M, Del Vecchio M, Gogas HJ, Arance AM, Cowey CL, Dalle S, Schenker M, Chiarion-Sileni V, Marquez-Rodas I, et al; CheckMate 238 Collaborators: Adjuvant nivolumab versus ipilimumab in resected stage III or IV melanoma. N Engl J Med 377: 1824-1835, 2017.

35. Eggermont AMM, Blank CU, Mandala M, Long GV, Atkinson V, Dalle S, Haydon A, Lichinitser M, Khattak A, Carlino MS, et al: Adjuvant pembrolizumab versus placebo in resected stage III melanoma. N Engl J Med 378: 1789-1801, 2018.

36. Larkin J, Chiarion-Sileni V, Gonzalez R, Grob JJ, Cowey CL, Lao CD, Schadendorf D, Dummer R, Smylie M, Rutkowski $\mathrm{P}$, et al: Combined nivolumab and ipilimumab or monotherapy in untreated melanoma. N Engl J Med 373: 23-34, 2015.

37. Larkin J, Chiarion-Sileni V, Gonzalez R, Grob JJ, Rutkowski P, Lao CD, Cowey CL, Schadendorf D, Wagstaff J, Dummer R, et al: Five-year survival with combined nivolumab and ipilimumab in advanced melanoma. N Engl J Med 381: 1535-1546, 2019.

38. Kirchberger MC, Hauschild A, Schuler G and Heinzerling L: Combined low-dose ipilimumab and pembrolizumab after sequential ipilimumab and pembrolizumab failure in advanced melanoma. Eur J Cancer 65: 182-184, 2016.

39. Long GV, Atkinson V, Cebon JS, Jameson MB, Fitzharris BM, McNeil CM, Hill AG, Ribas A, Atkins MB, Thompson JA, et al: Standard-dose pembrolizumab in combination with reduced-dose ipilimumab for patients with advanced melanoma (KEYNOTE-029): An open-label, phase 1b trial. Lancet Oncol 18: 1202-1210, 2017.
40. Zimmer L, Apuri S, Eroglu Z, Kottschade LA, Forschner A, Gutzmer R, Schlaak M, Heinzerling L, Krackhardt AM, Loquai $\mathrm{C}$, et al: Ipilimumab alone or in combination with nivolumab after progression on anti-PD-1 therapy in advanced melanoma. Eur J Cancer 75: 47-55, 2017.

41. Long GV, Robert C, Blank C, Ribas A, Mortier L, Schachter J, Middleton MR, et al: Outcomes in patients treated with ipilimumab after pembrolizumab in KEYNOTE-006. Eur J Cancer 72: S128-S129, 2017.

42. Ascierto PA, Butterfield LH, Demaria S, Ferris RL, Freeman GJ, Lo RS, Mantovani A, Nathan P, Hamid O, Politi K, et al: The great debate at 'Immunotherapy Bridge 2018', Naples, November 29th, 2018. J Immunother Cancer 7: 221, 2019.

43. Tawbi HA, Forsyth PA, Algazi A, Hamid O, Hodi FS, Moschos SJ, Khushalani NI, Lewis K, Lao CD, Postow MA, et al: Combined nivolumab and ipilimumab in melanoma metastatic to the brain. N Engl J Med 379: 722-730, 2018.

44. Long GV, Atkinson V, Lo S, Sandhu S, Guminski AD, Brown MP, Wilmott JS, Edwards J, Gonzalez M, Scolyer RA, et al: Combination nivolumab and ipilimumab or nivolumab alone in melanoma brain metastases: A multicentre randomised phase 2 study. Lancet Oncol 19: 672-681, 2018.

45. Conry RM, Westbrook B, McKee S and Norwood TG: Talimogene laherparepvec: First in class oncolytic virotherapy. Hum Vaccin Immunother 14: 839-846, 2018.

46. Andtbacka RH, Kaufman HL, Collichio F, Amatruda T, Senzer N, Chesney J, Delman KA, Spitler LE, Puzanov I, Agarwala SS, et al: Talimogene laherparepvec improves durable response rate in patients with advanced melanoma. J Clin Oncol 33: 2780-2788, 2015 .

47. Chesney J, Puzanov I, Collichio F, Singh P, Milhem MM, Glaspy J, Hamid O, Ross M, Friedlander P, Garbe C, et al: Randomized, open-label phase II study evaluating the efficacy and safety of talimogene laherparepvec in combination with ipilimumab versus ipilimumab alone in patients with advanced, unresectable melanoma. J Clin Oncol 36: 1658-1667, 2018.

48. Ribas A, Dummer R, Puzanov I, Vander Walde A, Andtbacka RHI, Michielin O, Olszanski AJ, Malvehy J, Cebon J, Fernandez E, et al: Oncolytic virotherapy promotes intratumoral $\mathrm{T}$ cell infiltration and improves anti-PD-1 immunotherapy. Cell 174: 1031-1032, 2018

49. Durham NM, Nirschl CJ, Jackson CM, Elias J, Kochel CM, Anders RA and Drake CG: Lymphocyte activation gene 3 (LAG-3) modulates the ability of CD4 T-cells to be suppressed in vivo. PLoS One 9: e109080, 2014

50. Andrews LP, Marciscano AE, Drake CG and Vignali DA: LAG3 (CD223) as a cancer immunotherapy target. Immunol Rev 276: 80-96, 2017.

51. Woo SR, Turnis ME, Goldberg MV, Bankoti J, Selby M, Nirschl CJ, Bettini ML, Gravano DM, Vogel P, Liu CL, et al: Immune inhibitory molecules LAG-3 and PD-1 synergistically regulate T-cell function to promote tumoral immune escape. Cancer Res 72: 917-927, 2012.

52. Ascierto PA, Bono P, Bhatia S, Melero I, Nyakas MS, Svane I, Larkin J, Gomez-Roca C, Schadendorf D, Dummer R, et al: LBA18Efficacy of BMS-986016, a monoclonal antibody that targets lymphocyte activation gene-3 (LAG-3), in combination with nivolumab in pts with melanoma who progressed during prior anti-PD-1/PD-L1 therapy (mel prior IO) in all-comer and biomarker-enriched populations. Ann Oncol 28 (Suppl 5): v605-v649, 2017

53. Das M, Zhu C and Kuchroo VK: Tim-3 and its role in regulating anti-tumor immunity. Immunol Rev 276: 97-111, 2017.

54. Ricciuti B, Leonardi GC, Puccetti P, Fallarino F, Bianconi V, Sahebkar A, Baglivo S, Chiari R and Pirro M: Targeting indoleamine-2,3-dioxygenase in cancer: Scientific rationale and clinical evidence. Pharmacol Ther 196: 105-116, 2019.

55. Long GV, Dummer R, Hamid O, Gajewski TF, Caglevic C, Dalle S, Arance A, Carlino MS, Grob JJ, Kim TM, et al: Epacadostat plus pembrolizumab versus placebo plus pembrolizumab in patients with unresectable or metastatic melanoma (ECHO-301/KEYNOTE-252): A phase 3, randomised, double-blind study. Lancet Oncol 20: 1083-1097, 2019.

56. Nicholas $C$ and Lesinski GB: Immunomodulatory cytokines as therapeutic agents for melanoma. Immunotherapy 3: 673-690, 2011.

57. Charych DH, Hoch U, Langowski JL, Lee SR, Addepalli MK, Kirk PB, Sheng D, Liu X, Sims PW, VanderVeen LA, et al: NKTR-214, an engineered cytokine with biased IL2 receptor binding, increased tumor exposure, and marked efficacy in mouse tumor models. Clin Cancer Res 22: 680-690, 2016. 
58. Diab A, Haymaker C, Bernatchez C, Andtbacka RHI, Shaheen M, Johnson D, Markowitz J, Puzanov I, Murthy R, Johnson DH, et al: Intratumoral (it) injection of the TLR9 agonist tilsotolimod (imo-2125) in combination with ipilimumab (ipi) triggers durable responses in pd-1 inhibitor refractory metastatic melanoma $(\mathrm{rmm})$ : results from a multicenter, phase $1 / 2$ study. Ann Oncol 29 (Suppl 8): viii442-viii466, 2018.

59. Milhem MM, Long GV, Hoimes CJ, Amin A, Lao CD, Conry RM, Hunt J, Daniels GA, Almubarak M, Shaheen MF, et al: Phase 1b/2, open label, multicenter, study of the combination of SD-101 and pembrolizumab in patients with advanced melanoma who are naïve to anti-PD-1 therapy. J Clin Oncol 37 (Suppl 15): 9534-9534, 2019.

60. Wiehagen KR, Girgis NM, Yamada DH, Smith AA, Chan SR, Grewal IS, Quigley M and Verona RI: Combination of CD40 agonism and CSF-1R blockade reconditions tumor-associated macrophages and drives potent antitumor immunity. Cancer Immunol Res 5: 1109-1121, 2017.

61. Mantovani A, Marchesi F, Malesci A, Laghi L and Allavena P: Tumour-associated macrophages as treatment targets in oncology. Nat Rev Clin Oncol 14: 399-416, 2017.

62. Cannarile MA, Weisser M, Jacob W, Jegg AM, Ries CH and Rüttinger D: Colony-stimulating factor 1 receptor (CSF1R) inhibitors in cancer therapy. J Immunother Cancer 5: 53, 2017.

63. Sarnaik AA, Yu B, Yu D, Morelli D, Hall M, Bogle D, Yan L, Targan S, Solomon J, Nichol G, et al: Extended dose ipilimumab with a peptide vaccine: Immune correlates associated with clinical benefit in patients with resected high-risk stage IIIc/IV melanoma. Clin Cancer Res 17: 896-906, 2011.

64. Ribas A, Comin-Anduix B, Chmielowski B, Jalil J, de la Rocha P, McCannel TA, Ochoa MT, Seja E, Villanueva A, Oseguera DK, et al: Dendritic cell vaccination combined with CTLA4 blockade in patients with metastatic melanoma. Clin Cancer Res 15: 6267-6276, 2009.

65. Wilgenhof S, Van Nuffel AM, Benteyn D, Corthals J, Aerts C, Heirman C, Van Riet I, Bonehill A, Thielemans K and Neyns B: A phase IB study on intravenous synthetic mRNA electroporated dendritic cell immunotherapy in pretreated advanced melanoma patients. Ann Oncol 24: 2686-2693, 2013.

66. Wilgenhof S, Corthals J, Heirman C, van Baren N, Lucas S, Kvistborg P, Thielemans K and Neyns B: Phase II study of autologous monocyte-derived mRNA electroporated dendritic cells (TriMixDC-MEL) plus ipilimumab in patients with pretreated advanced melanoma. J Clin Oncol 34: 1330-1338, 2016.

67. Chick RC, Faries MB, Hale DF, Kemp Bohan PM, Hickerson A Vreelande TJ, Myers JW, Cindass JL, Brown TA, et al: Multi-institutional, prospective, randomized, double-blind, placebo-controlled phase IIb trial of the tumor lysate, particle-loaded, dendritic cell (TLPLDC) vaccine to prevent recurrence in high-risk melanoma patients: A subgroup analysis. J Clin Oncol 38 (Suppl 5): 63-63, 2020.

68. Kvistborg P, Philips D, Kelderman S, Hageman L, Ottensmeier C, Joseph-Pietras D, Welters MJ, van der Burg S, Kapiteijn E, Michielin O, et al: Anti-CTLA-4 therapy broadens the melanoma-reactive $\mathrm{CD}^{+} \mathrm{T}$ cell response. Sci Transl Med 6 : 254ra128, 2014.

69. Daud AI, Loo K, Pauli ML, Sanchez-Rodriguez R, Sandoval PM, Taravati K, Tsai K, Nosrati A, Nardo L, Alvarado MD, et al: Tumor immune profiling predicts response to anti-PD-1 therapy in human melanoma. J Clin Invest 126: 3447-3452, 2016.

70. Foley KC, Nishimura MI and Moore TV: Combination immunotherapies implementing adoptive T-cell transfer for advanced-stage melanoma. Melanoma Res 28: 171-184, 2018

71. Rosenberg SA, Packard BS, Aebersold PM, Solomon D, Topalian SL, Toy ST, Simon P, Lotze MT, Yang JC, Seipp CA, et al: Use of tumor-infiltrating lymphocytes and interleukin-2 in the immunotherapy of patients with metastatic melanoma. A preliminary report. N Engl J Med 319: 1676-1680, 1988.

72. Rosenberg SA, Yang JC, Sherry RM, Kammula US, Hughes MS, Phan GQ, Citrin DE, Restifo NP, Robbins PF, Wunderlich JR, et al: Durable complete responses in heavily pretreated patients with metastatic melanoma using T-cell transfer immunotherapy. Clin Cancer Res 17: 4550-4557, 2011

73. Feins S, Kong W, Williams EF, Milone MC and Fraietta JA: An introduction to chimeric antigen receptor (CAR) T-cell immunotherapy for human cancer. Am J Hematol 94: S3-S9, 2019.

74. Robbins PF, Kassim SH, Tran TL, Crystal JS, Morgan RA, Feldman SA, Yang JC, Dudley ME, Wunderlich JR, Sherry RM, et al A pilot trial using lymphocytes genetically engineered with an NY-ESO-1-reactive T-cell receptor: Long-term follow-up and correlates with response. Clin Cancer Res 21: 1019-1027, 2015.
75. Falzone L, Salomone S and Libra M: Evolution of cancer pharmacological treatments at the turn of the third millennium. Front Pharmacol 9: 1300, 2018

76. Pelster MS and Amaria RN: Combined targeted therapy and immunotherapy in melanoma: A review of the impact on the tumor microenvironment and outcomes of early clinical trials. Ther Adv Med Oncol 11: 1758835919830826, 2019.

77. Ribas A, Hodi FS, Callahan M, Konto C and Wolchok J: Hepatotoxicity with combination of vemurafenib and ipilimumab. N Engl J Med 368: 1365-1366, 2013.

78. Minor DR, Puzanov I, Callahan MK, Hug BA and Hoos A: Severe gastrointestinal toxicity with administration of trametinib in combination with dabrafenib and ipilimumab. Pigment Cell Melanoma Res 28: 611-612, 2015.

79. Ribas A, Butler M, Lutzky J, Lawrence DP, Robert C, Miller W, Linette WMGP, Ascierto PA, Kuzel T, Algazi AP, et al: Phase I study combining anti-PD-L1 (MEDI4736) with BRAF (dabrafenib) and/or MEK (trametinib) inhibitors in advanced melanoma. J Clin Oncol 33 (Suppl 15): 3003, 2015.

80. Shui L, Yang X, Li J, Yi C, Sun Q and Zhu H: Gut microbiome as a potential factor for modulating resistance to cancer immunotherapy. Front Immunol 10: 2989, 2020.

81. Longo V, Brunetti O, Azzariti A, Galetta D, Nardulli P, Leonetti F and Silvestris N: Strategies to improve cancer immune checkpoint inhibitors efficacy, other than abscopal effect: A systematic review. Cancers (Basel) 11: 539, 2019.

82. Velez MA, Burns TF and Stabile LP: The estrogen pathway as a modulator of response to immunotherapy. Immunotherapy 11: 1161-1176, 2019.

83. Matsushita $\mathrm{M}$ and Kawaguchi M: Immunomodulatory Effects of Drugs for Effective Cancer Immunotherapy. J Oncol 2018: 8653489, 2018.

84. Vivarelli S, Falzone L, Basile MS, Nicolosi D, Genovese C, Libra $\mathrm{M}$ and Salmeri M: Benefits of using probiotics as adjuvants in anticancer therapy (Review). World A Sci J 1: 125-135, 2019.

85. Sivan A, Corrales L, Hubert N, Williams JB, Aquino-Michaels K, Earley ZM, Benyamin FW, Lei YM, Jabri B, Alegre ML, et al: Commensal Bifidobacterium promotes antitumor immunity and facilitates anti-PD-L1 efficacy. Science 350: 1084-1089, 2015

86. Routy B,LeChatelierE,DerosaL,Duong CPM,AlouMT,Daillère R, Fluckiger A, Messaoudene M, Rauber C, Roberti MP, et al: Gut microbiome influences efficacy of PD-1-based immunotherapy against epithelial tumors. Science 359: 91-97, 2018.

87. Gopalakrishnan V, Spencer CN, Nezi L, Reuben A, Andrews MC, Karpinets TV, Prieto PA, Vicente D, Hoffman K, Wei SC, et al: Gut microbiome modulates response to anti-PD-1 immunotherapy in melanoma patients. Science 359: 97-103, 2018.

88. Banna GL, Torino F, Marletta F, Santagati M, Salemi R, Cannarozzo E, Falzone L, Ferraù F and Libra M: Lactobacillus rhamnosus GG: An overview to explore the rationale of its use in cancer. Front Pharmacol 8: 603, 2017.

89. Vétizou M, Pitt JM, Daillère R, Lepage P, Waldschmitt N, Flament C, Rusakiewicz S, Routy B, Roberti MP, Duong CP, et al: Anticancer immunotherapy by CTLA-4 blockade relies on the gut microbiota. Science 350: 1079-1084, 2015.

90. Matson V, Fessler J, Bao R, Chongsuwat T, Zha Y, Alegre ML, Luke JJ and Gajewski TF: The commensal microbiome is associated with anti-PD-1 efficacy in metastatic melanoma patients. Science 359: 104-108, 2018.

91. Elkrief A, Derosa L, Kroemer G, Zitvogel L and Routy B: The negative impact of antibiotics on outcomes in cancer patients treated with immunotherapy: A new independent prognostic factor? Ann Oncol 30: 1572-1579, 2019.

92. Eskiocak B, McMillan EA, Mendiratta S, Kollipara RK, Zhang H, Humphries CG, Wang C, Garcia-Rodriguez J, Ding M, Zaman A, et al: Biomarker accessible and chemically addressable mechanistic subtypes of BRAF melanoma. Cancer Discov 7: 832-851, 2017.

93. Salemi R, Falzone L, Madonna G, Polesel J, Cinà D, Mallardo D, Ascierto PA, Libra M and Candido S: MMP-9 as a candidate marker of response to BRAF inhibitors in melanoma patients with BRAFV600E mutation detected in circulating-free DNA. Front Pharmacol 9: 856, 2018.

94. Guarneri C, Bevelacqua V, Polesel J, Falzone L, Cannavò PS, Spandidos DA, Malaponte G and Libra M: NFKB inhibition is associated with OPN/MMP 9 downregulation in cutaneous melanoma. Oncol Rep 37: 737-746, 2017.

95. Luke JJ, Flaherty KT, Ribas A and Long GV: Targeted agents and immunotherapies: Optimizing outcomes in melanoma. Nat Rev Clin Oncol 14: 463-482, 2017. 
96. Tumeh PC, Harview CL, Yearley JH, Shintaku IP, Taylor EJ, Robert L, Chmielowski B, Spasic M, Henry G, Ciobanu V, et al: PD-1 blockade induces responses by inhibiting adaptive immune resistance. Nature 515: 568-571, 2014.

97. Gajewski TF, Louahed J and Brichard VG: Gene signature in melanoma associated with clinical activity: A potential clue to unlock cancer immunotherapy. Cancer J 16: 399-403, 2010.

98. Harlin H, Meng Y, Peterson AC, Zha Y, Tretiakova M, Slingluff C, McKee $M$ and Gajewski TF: Chemokine expression in melanoma metastases associated with $\mathrm{CD} 8^{+} \mathrm{T}$-cell recruitment. Cancer Res 69: 3077-3085, 2009.

99. Ji RR, Chasalow SD, Wang L, Hamid O, Schmidt H, Cogswell J, Alaparthy S, Berman D, Jure-Kunkel M, Siemers NO, et al: An immune-active tumor microenvironment favors clinical response to ipilimumab. Cancer Immunol Immunother 61: 1019-1031, 2012.

100. Spranger S, Bao R and Gajewski TF: Melanoma-intrinsic $\beta$-catenin signalling prevents anti-tumour immunity. Nature 523 231-235, 2015

101. Peng W, Chen JQ, Liu C, Malu S, Creasy C, Tetzlaff MT, Xu C, McKenzie JA, Zhang C, Liang X, et al: Loss of PTEN promotes resistance to T cell-mediated immunotherapy. Cancer Discov 6: 202-216, 2016.

102.Zaretsky JM, Garcia-Diaz A, Shin DS, Escuin-Ordinas H, Hugo W, Hu-Lieskovan S, Torrejon DY, Abril-Rodriguez G, Sandoval S, Barthly L, et al: Mutations associated with acquired resistance to PD 1 blockade in melanoma. N Engl J Med 375 819-829, 2016.

103. Shin DS, Zaretsky JM, Escuin-Ordinas H, Garcia-Diaz A, Hu-Lieskovan S, Kalbasi A, Grasso CS, Hugo W, Sandoval S, Torrejon DY, et al: Primary resistance to PD 1 blockade mediated by JAK1/2 mutations. Cancer Discov 7: 188-201, 2017.
104. Liu D, Schilling B,Liu D, Sucker A,Livingstone E, Jerby-Arnon L, Zimmer L, Gutzmer R, Satzger I, Loquai C, et al: Integrative molecular and clinical modeling of clinical outcomes to PD1 blockade in patients with metastatic melanoma. Nat Med 25: 1916-1927, 2019.

105. Tuaeva NO, Falzone L, Porozov YB, Nosyrev AE, Trukhan VM, Kovatsi L, Spandidos DA, Drakoulis N, Kalogeraki A, Mamoulakis C, et al: Translational application of circulating DNA in oncology: review of the last decades achievements. Cells 8: 1251, 2019.

106. Lee EY and Kulkarni RP: Circulating biomarkers predictive of tumor response to cancer immunotherapy. Expert Rev Mol Diagn 19: 895-904, 2019

107. Napoli S, Scuderi C, Gattuso G, Bella VD, Candido S, Basile MS, Libra $\mathrm{M}$ and Falzone L: Functional roles of matrix metalloproteinases and their inhibitors in melanoma. Cells 9: E1151, 2020.

108. Falzone L, Salemi R, Travali S, Scalisi A, McCubrey JA, Candido S and Libra M: MMP-9 overexpression is associated with intragenic hypermethylation of MMP9 gene in melanoma. Aging (Albany NY) 8: 933-944, 2016.

109. Moogk D, da Silva IP, Ma MW, Friedman EB, de Miera EV, Darvishian F, Scanlon P, Perez-Garcia A, Pavlick AC, Bhardwaj N, et al: Melanoma expression of matrix metalloproteinase-23 is associated with blunted tumor immunity and poor responses to immunotherapy. J Transl Med 12: 342, 2014.

(i) () $\Theta$ This work is licensed under a Creative Commons International (CC BY-NC-ND 4.0) License. 